\title{
Fermi hypernetted-chain study of half-filled Landau levels with broken rotational symmetry
}

\author{
Orion Ciftja and Carlos Wexler \\ Department of Physics and Astronomy, University of Missouri-Columbia, Columbia, Missouri 65211
}

(Received 7 November 2001; published 26 April 2002)

\begin{abstract}
We investigate broken rotational symmetry (BRS) states at half-filling of the valence Landau level (LL). We generalize Rezayi and Read's (RR) trial wave function, a special case of Jain's composite fermion (CF) wave functions, to include anisotropic coupling of the flux quanta to electrons, thus generating a nematic order in the underlying CF liquid. Using the Fermi hypernetted-chain method, which readily gives results in the thermodynamic limit, we determine the properties of these states in detail. By using the anisotropic pair distribution and static structure functions we determine the correlation energy and find that, as expected, RR's state is stable in the lowest LL, whereas BRS states may occur at half-filling of higher LL's, with a possible connection to the recently discovered quantum Hall liquid crystals.
\end{abstract}

DOI: 10.1103/PhysRevB.65.205307

PACS number(s): 73.43.-f, 64.70.Md

\section{INTRODUCTION}

During the past two decades the physics of twodimensional electron systems (2DES's) has provided some of the richest grounds for developments in condensed-matter physics. In particular the integer ${ }^{1}$ and fractional $^{2}$ quantum Hall effects in strong magnetic fields are some of the most remarkable phenomena discovered in the second half of the 20th century, and rival superconductivity in their fundamental significance by manifesting quantum mechanics on macroscopic scales and providing a major impetus to the development of ideas in many-body physics, ${ }^{3}$ like the existence of fractionally charged quasiparticles, ${ }^{4}$ topological quantum numbers, ${ }^{5}$ chiral Luttinger liquids, ${ }^{6}$ composite particles, ${ }^{7}$ etc. One of the reasons that 2DES's keep supplying exciting results is the improved quality of the samples with mobilities increasing roughly exponentially with time, thus allowing the emergence of subtler effects due to electronic correlations (which are enhanced because of the reduced dimensionality).

Consider, initially, a 2DES at half-filling $(\nu=1 / 2)$ of the lowest Landau level (LLL). Experimentally, this state does not exhibit the typical features of the fractional quantum Hall effect (FQHE), namely, the very precise quantization of the transverse conductance in units of $e^{2} / h$, or the vanishing longitudinal resistivity. However, the resistivity shows a broad minimum, ${ }^{8}$ and an anomalous behavior in the propagation of surface acoustic waves ${ }^{9}$ has been observed. Early numerical work by Haldane ${ }^{10}$ suggested that $\nu=1 / 2$ is not incompressible. The overall experimental evidence strongly suggests that, in the LLL, for half-filling the system behaves like a strange Fermi liquid at close to zero effective magnetic field. ${ }^{11-13}$

A theory of compressible Fermi-liquid-like behavior at half-filling was proposed by Halperin, Lee, and Read: ${ }^{14}$ a two-dimensional (2D) system of electrons subjected to an external perpendicular magnetic field, at half-filling of the LLL, can be transformed to a mathematically equivalent system of fermions interacting with a Chern-Simons gauge field such that the average effective magnetic field acting on the fermions is zero. "Since these fermions do not "see" a net magnetic field, they can form a 2D Fermi sea of uniform density. In a very successful approach, based on the compos- ite fermion (CF) theory, ${ }^{7,15}$ Rezayi-Read ${ }^{16}$ (RR) described the half-filled state by a correlated Fermi wave function that is a product of a Slater determinant of plane waves, with a Jastrow factor corresponding to a Bose Laughlin state at half-filling,

$$
\Psi\left(\mathbf{r}_{1}, \ldots, \mathbf{r}_{N}\right)=\hat{P}_{0} \prod_{j<k}^{N}\left(z_{j}-z_{k}\right)^{2} e^{-\sum_{k=1}^{N}\left|z_{k}\right|^{2} / 4} \operatorname{det}\left[\varphi_{\mathbf{k}}\left(\mathbf{r}_{i}\right)\right]
$$

where $\varphi_{\mathbf{k}}\left(\mathbf{r}_{i}\right)$ are 2D plane waves for fully spin polarized CF states that fill a 2D disk in reciprocal space with Fermi momentum $k_{F}, z_{j}=x_{j}+i y_{j}$ is the complex $2 \mathrm{D}$ coordinate of $j$ th electron, and $\hat{P}_{0}$ is a projector onto the $\operatorname{LLL}(L=0)$. We work in units of the magnetic length $\left(l_{0}^{2}=\hbar / e B=1\right)$.

The situation is dramatically different for the next Landau level (LL) (LL index $L=1$ ), where for $\nu \simeq 5 / 2$ a quantized Hall conductance is observed along with a strong reduction of the longitudinal conductance, ${ }^{17}$ which are an indication of a Cooper pairing instability of the CF's. ${ }^{18}$ A discussion of this state is a wide and complex topic by itself, and this, along with the properties of the $\nu=3 / 2$ and $7 / 2$ states (halffilling of the upper-spin subband) will not be addressed in this work.

In this paper we discuss some aspects related to the many interesting phenomena that have recently emerged in nearly half-filled higher LL's (with LL index $L \geqslant 2$ ), in particular the extreme anisotropy measured in the low-temperature magnetotransport. ${ }^{19-21}$ This anisotropic behavior was attributed to the formation of a nematic phase of a 2DES which, at higher temperatures, undergoes a nematic to isotropic transition. $^{22,23}$ The motivation of our work is to study these nematic phases by means of many-body trial wave functions with broken rotation symmetry (BRS) at half-filling of a Landau level. Previously ${ }^{24}$ we reported on the existence of a BRS instability of the Laughlin state $^{4}$ for 1/3-filled higher LL's (with $L \geqslant 1$ ). Here we discuss a similar procedure for the more complex case of $1 / 2$ filling, which is more closely related to the experiments on anisotropic phases. ${ }^{19-21}$

Theoretically, Hartree-Fock (HF) calculations ${ }^{25-27}$ have been very succesful in predicting the anisotropic state in 
higher LL's and explaining the (re)orientation of the stripes when an in-plane magnetic field is applied. ${ }^{28}$ The HF theory suggests that for high LL's single-Slater determinant states of charge-density-wave (CDW) form have lower energies than Laughlin-type isotropic liquid states. These CDW states, with stripe order, which are predicted to be stable for partially filled LL's are, indeed, good candidates for the ground state at $L \geqslant 2$, although questions remain about whether are they stable against quantum fluctuations that in principle can produce liquid crystalline behavior. ${ }^{22,29}$ We note that the BRS states considered in this work are qualitatively different from CDW or Wigner crystal states because they represent a liquid crystalline state with nematic, rather than smectic or fully crystalline order.

Similarly to the Musaelian and Joynt's ${ }^{30}$ generalization of Laughlin's FQHE state ${ }^{4}$ used by us in Ref. 24, we add a symmetry breaking parameter $\alpha$ in the RR wave function [Eq. (1)]

$$
\begin{aligned}
\Psi_{\alpha}\left(\mathbf{r}_{1}, \ldots, \mathbf{r}_{N}\right)= & \hat{P}_{L} \prod_{j<k}^{N}\left(z_{j}-z_{k}+\alpha\right)\left(z_{j}-z_{k}-\alpha\right) \\
& \times e^{-\sum_{k=1}^{N}\left|z_{k}\right|^{2} / 4} \operatorname{det}\left[\varphi_{\mathbf{k}}\left(\mathbf{r}_{i}\right)\right],
\end{aligned}
$$

where $\hat{P}_{L}$ now represents a projector onto the $L$ th LL. This BRS wave function represents a homogeneous Fermi-liquidlike state at half-filling factor, and for $\alpha \neq 0$ has a nematic order (for $\alpha=0$ we recover the RR wave function which is obviously isotropic). Note that the magnitude of $\alpha$ is related to the amount of anisotropy, and its phase to the angle the director of the nematic (for real $\alpha$ the system will have a stronger modulation in the $x$ direction, and therefore likely have larger conductance in the perpendicular direction: $\sigma_{y y}$ $>\sigma_{x x}$ ). This wave function is an obvious starting point to study the nematic quantum Hall liquid crystals at half-filling, by facilitating the systematic study of the energy dependence of BRS states for diverse physical parameters (LL index, width of the 2DES, etc).

We study the BRS state for $1 / 2$ filling of the valence LL (i.e., $\nu=M+1 / 2$, with $M$ an integer) by using the Fermi hypernetted-chain (FHNC) method. ${ }^{31-35}$ This method allows us to compute physical quantities in the thermodynamic limit, without the limitations of using a finite number of particles that hinder other techniques, where the extrapolation of the results to the thermodynamic limit is not totally unambiguous.

We find that, for realistic interaction potentials, the RR (isotropic) state is stable in the LLL, whereas a BRS state is possible in higher LL's. While this is consistent with the view of BRS states for $L \geqslant 2$, it does not reflect the situation for $L=1$, where it is believed that CF's can form an incompressible state by a Bardeen-Cooper-Schrieffer-like pairing, ${ }^{36}$ as first pointed out by Moore and Read. ${ }^{37}$ Recent exact diagonalization results by Morf ${ }^{38}$ strongly suggest that such spin-polarized Pfaffian state is the best candidate to describe this filling.

In Sec. II we present the basic theoretical calculations needed to determine the stability of an isotropic or BRS state. A detailed description of the FHNC formalism in the context of the BRS wave function [Eq. (2)] is given in Sec. III. The results for the BRS state in the LLL and their extension in higher LL's are discussed in Sec. IV.

\section{BASIC THEORY}

In this work we study the stability of different states by using trial wave functions of the form of Eq. (2). We perform this analysis by comparing the energy in each of these states to find the optimum value for the anisotropy-generating parameter $\alpha$. The potential, or correlation energy per electron is given by

$$
E_{\alpha}=\frac{1}{N} \frac{\left\langle\Psi_{\alpha}|\hat{V}| \Psi_{\alpha}\right\rangle}{\left\langle\Psi_{\alpha} \mid \Psi_{\alpha}\right\rangle}=\frac{\rho}{2} \int d^{2} r V(r)[g(\mathbf{r})-1],
$$

where $\hat{V}$ represents the electron-electron, electronbackground, and background-background interaction; and $g(\mathbf{r})$ is the (angle-dependent) pair distribution function given by

$$
g(\mathbf{r})=\frac{N(N-1)}{\rho^{2}} \frac{\int d^{2} r_{3} \cdots d^{2} r_{N}\left|\Psi_{\alpha}\left(\mathbf{r}_{1} \cdots \mathbf{r}_{N}\right)\right|^{2}}{\int d^{2} r_{1} \cdots d^{2} r_{N}\left|\Psi_{\alpha}\left(\mathbf{r}_{1} \cdots \mathbf{r}_{N}\right)\right|^{2}},
$$

where $\mathbf{r}=\mathbf{r}_{2}-\mathbf{r}_{1}$. The following normalization condition, $\rho \int d^{2} r[g(\mathbf{r})-1]=-1$, can be used as a convenient check for numerical procedures. For an ideal 2D sample the interaction is a pure Coulomb potential $V(r) \simeq e^{2} /(\epsilon r)$, while in samples with finite thickness a reasonable choice is the Zhang-Das Sarma (ZDS) potential ${ }^{39} V(r)=e^{2} /\left(\epsilon \sqrt{r^{2}+\lambda^{2}}\right)$, where $\lambda$ is of the order of the sample thickness. Alternatively, the correlation energy can be computed in reciprocal space,

$$
E_{\alpha}=\frac{1}{2} \int \frac{d^{2} q}{(2 \pi)^{2}} \widetilde{V}(q)[S(\mathbf{q})-1]
$$

where $\widetilde{V}(q)$ is the 2D Fourier transform ${ }^{40}$ (FT) of $V(r)$ and $S(\mathbf{q})$ is the static structure factor:

$$
S(\mathbf{q})-1=\rho F T[g(\mathbf{r})-1] .
$$

While both $g(\mathbf{r})$ and $S(\mathbf{q})$ are angle-dependent (e.g., see Figs. 1 and 2), because the interaction potential is centrally symmetric, the energy $E_{\alpha}$ depends only on the angleaveraged pair distribution function or static structure factor defined as

$$
\bar{g}(r)=\int_{0}^{2 \pi} \frac{d \theta}{2 \pi} g(\mathbf{r}), \quad \bar{S}(q)=\int_{0}^{2 \pi} \frac{d \theta_{q}}{2 \pi} S(\mathbf{q}) .
$$

The determination of either the pair distribution function or the structure factor is generally a complicated integral problem that needs to be solved for each LL. However, its is known that if transitions to other LL's are neglected (i.e., a single-LL approximation), $g(\mathbf{r})$ and $S(\mathbf{q})$ at higher LL's are simply related to those at the LLL, $(L=0)$ by means of a 
(a)

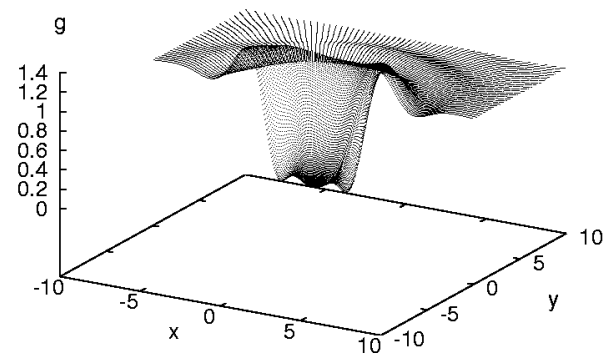

(c)

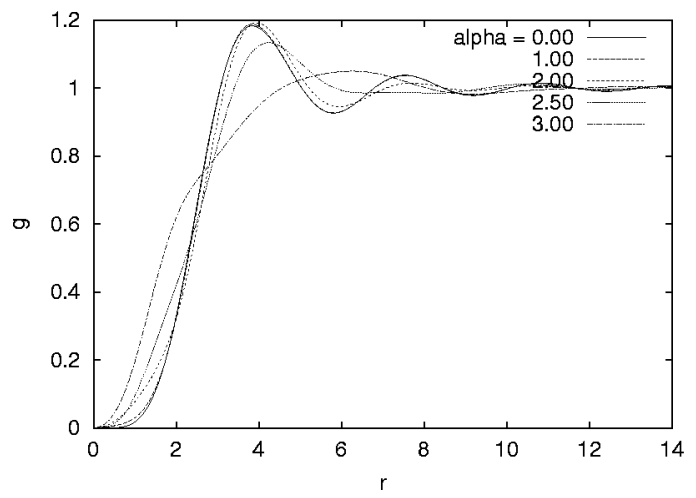

(b)

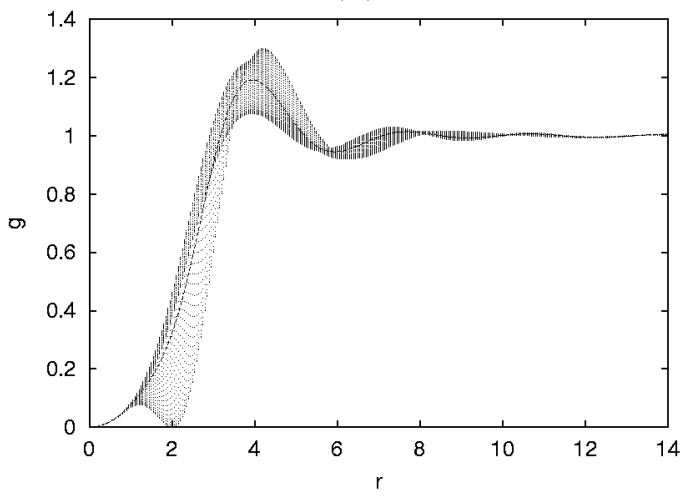

(d)

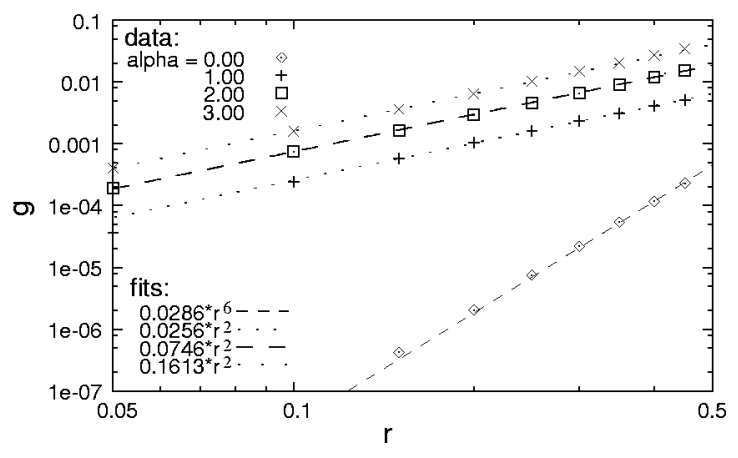

FIG. 1. Pair distribution function for the BRS state at $\nu=1 / 2$. (a) $\alpha=2$, surface plot of $g$ (r) (the surface for $y<0$ was removed for clarity). (b) $\alpha=2$; dotted lines: $g(r, \theta)$ for various $\theta \in[0,2 \pi]$; full line: angle-averaged $\bar{g}(r)$. (c) Angle-averaged $\bar{g}(r)$ for $\alpha=0,1,2,2.5$, and 3. (d) Small- $r$ behavior of $\bar{g}(r)$; lines are fitting curves. Note the discrete nodes of $g(r, \theta)$ at $r=\alpha, \theta=\theta_{\alpha}, \theta_{\alpha}+\pi\left(\theta_{\alpha}=0\right.$ in this case). Calculations were performed in the $\mathrm{FHNC} / 0$ approximation.

convolution or product respectively. We will apply this approximation (which, moreover, quenches the kinetic energy in higher LL's as well). It is then, sufficient to compute these distribution functions once in the LLL, and then the correlation energy per electron is given by

$$
E_{\alpha}^{L}=\frac{1}{2} \int \frac{d^{2} q}{(2 \pi)^{2}} \widetilde{V}_{\mathrm{eff}}(q)[S(\mathbf{q})-1],
$$

where $\widetilde{V}_{\text {eff }}(q) \equiv \widetilde{V}(q)\left[L_{L}\left(q^{2} / 2\right)\right]^{2} . L_{L}(z)$ are Laguerre polynomials, and $S(\mathbf{q})$ is calculated in the $\operatorname{LLL}(L=0)$. In what follows we compute $g(\mathbf{r})$ and $S(\mathbf{q})$ using the FHNC method.

\section{FERMI HNC METHOD FOR THE BROKEN ROTATIONAL SYMMETRY STATE}

The development of the FHNC method for Fermi systems $^{31,32}$ allows one to estimate accurately the expectation value of a Hamiltonian, the pair distribution function and related quantities associated with a Jastrow-Slater wave function and other more complex many-body Fermi wave functions. The FHNC method treats the correlated system of particles a priori in the thermodynamic limit, and therefore is extremely useful in the study of infinite homogeneous
Fermi systems described by correlated Fermi wave functions, having (but not limited to) a Jastrow-Slater form. In addition one can prove that the FHNC scheme achieves convergence of the expectation value of a Hamiltonian within some expansion scheme, ${ }^{41}$ thus substantiating the belief that the FHNC gives an accurate upper bound for the energy and other related quantities. ${ }^{41,42}$

An important problem arises at this stage. The projection onto the $L$ th $L L$ performed in the RR wave function or its generalization [Eq. (2)] leads to a wave function that cannot be directly treated within the FHNC formalism, because the simple Jastrow $\times$ Slater determinant structure of single particle orbitals is lost. We therefore use an unprojected version of Eq. (2), which, although approximate, is believed to contain the most important physics, especially since the Jastrow factors already significantly annihilate higher-LL components of the wave function. ${ }^{43}$ In addition, although the wave function given in Eq. (2) has a Jastrow-Slater form after dropping the projector $P_{L}$, the FHNC method in this case differs from the standard approach since two-body correlation factors and related quantities depend not only on interparticle distance, but also on the relative angle between the particles. In order to calculate the pair distribution function the modulus square of the wave function, 
(a)

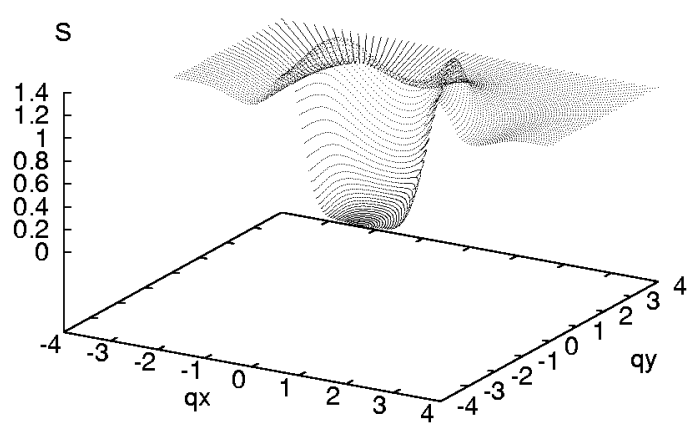

(b)

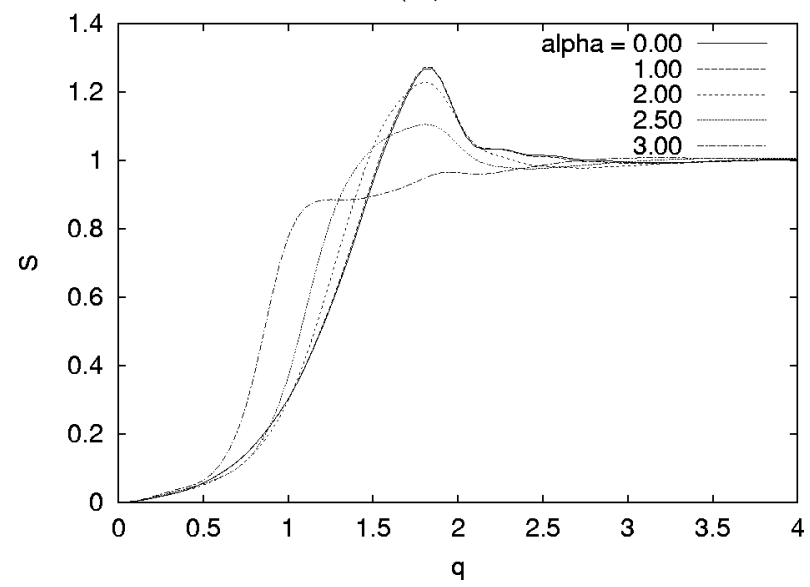

FIG. 2. Static structure factor for the BRS state at $\nu=1 / 2$. (a) $\alpha=2$, surface plot of $S(\mathbf{q})$ (the surface for $q_{y}<0$ was removed for clarity). (b) Angle-averaged $\bar{S}(q)$ for $\alpha=0,1,2,2.5$, and 3. Note the presence of peaks in $S(\mathbf{q})$, consistent with a nematic structure. Calculations were performed in the FHNC/0 approximation.

$$
\begin{aligned}
\left|\Psi_{\alpha}\left(\mathbf{r}_{1}, \ldots, \mathbf{r}_{N}\right)\right|^{2}= & e \sum_{i>j}^{N} u\left(z_{i}-z_{j}\right) \\
& \times e^{-\sum_{i=1}^{N}\left(\left|z_{i}\right|^{2} / 2\right)}\left|\operatorname{det}\left[\varphi_{\mathbf{k}}\left(\mathbf{r}_{i}\right)\right]\right|^{2},
\end{aligned}
$$

where $u(z)=\ln |z-\alpha|^{2}+\ln |z+\alpha|^{2}$, is expanded perturbatively in terms of $h\left(\mathbf{r}_{i j}\right)=\exp \left[u\left(\mathbf{r}_{i j}\right)\right]-1$, and may be ordered as

$$
\begin{aligned}
\left|\Psi_{\alpha}\right|^{2}= & {\left[1+\sum_{i<j}^{N} h\left(\mathbf{r}_{i j}\right)+\sum_{i<j}^{N} \sum_{k<l}^{N} h\left(\mathbf{r}_{i j}\right) h\left(r_{k l}\right)+\cdots\right] } \\
& \times\left|\operatorname{det}\left[\varphi_{\mathbf{k}}\left(\mathbf{r}_{i}\right)\right]\right|^{2} .
\end{aligned}
$$

In addition to dynamical correlations between particles, described by the pseudopotential $u\left(z_{i}-z_{j}\right)$, there are also statistical correlations described by the Slater determinant which renders the whole state antisymmetric. Similar to the Jastrow part, we may expand $\left|\operatorname{det}\left[\varphi_{\mathbf{k}}\left(\mathbf{r}_{i}\right)\right]\right|^{2}$ in the number of exchanges between particles. In this way Eq. (10) becomes a quite symmetrical expansion in the number of dynamical correlation factors, $h\left(\mathbf{r}_{i j}\right)$, as well as the number of statistical correlation factors. The resulting cluster terms in the expres- sion for $g\left(\mathbf{r}_{12}\right)$ contain both kinds of correlations, and may be represented by cluster diagrams. As in the Bose case, the associated pair distribution function $g\left(\mathbf{r}_{12}\right)$ is then given by the sum of all linked irreducible diagrams obeying welldefined topological rules. ${ }^{31}$

One defines nodal, composite (non-nodal), and elementary diagrams as in the Bose case, but there are now four different types for each of them. The four different classes of nodal, composite, and elementary diagrams are generally denoted by dd (direct-direct), de (direct-exchange), ee (exchange-exchange), and cc (circular-exchange). Then the pair distribution function is obtained from the set of FHNC equations given in the Appendix, where $\rho$ is the density, $g_{s}(=1)$ is the spin degeneracy, and $l\left(r_{12}\right)$ is the familiar statistical exchange factor for the 2D Slater determinant given by

$$
l\left(\mathbf{r}_{1}, \mathbf{r}_{2}\right)=2 \frac{J_{1}\left(k_{F} r_{12}\right)}{k_{F} r_{12}},
$$

where $r_{12}=\left|\mathbf{r}_{2}-\mathbf{r}_{1}\right|$, and $J_{1}(x)$ is the first order Bessel function.

For standard systems like the ${ }^{3} \mathrm{He}$ Fermi liquid, the pair correlation factor is short ranged, and heals to 1 for large distances; therefore, the function $\exp \left[u\left(\mathbf{r}_{i j}\right)\right]-1$ provides a possible expansion parameter [note that in order to apply the Fermi HNC expansion, the correlation (pseudo)potential has to satisfy the conditions: $u\left(\mathbf{r}_{i j} \rightarrow 0\right) \rightarrow-\infty$ and $u\left(\mathbf{r}_{i j} \rightarrow+\infty\right)$ $\rightarrow 0]$. In the case of the BRS wave function, the (pseudo) potential $u(\mathbf{r})$ is logarithmically long ranged; however, formally it is possible to extend the method by splitting the pseudopotential associated with the Jastrow part into shortand long-ranged parts, respectively,

$$
u\left(\mathbf{r}_{12}\right)=u_{s}\left(\mathbf{r}_{12}\right)+u_{l}\left(\mathbf{r}_{12}\right),
$$

with the dd nodal and composite function similarly split:

$$
\begin{aligned}
& N_{d d}\left(\mathbf{r}_{12}\right)=N_{d d s}\left(\mathbf{r}_{12}\right)-u_{l}\left(\mathbf{r}_{12}\right), \\
& X_{d d}\left(\mathbf{r}_{12}\right)=X_{d d s}\left(\mathbf{r}_{12}\right)+u_{l}\left(\mathbf{r}_{12}\right) .
\end{aligned}
$$

The splitting is done subject to the conditions

$$
\begin{gathered}
u\left(\mathbf{r}_{12}\right)+N_{d d}\left(\mathbf{r}_{12}\right)=u_{s}\left(\mathbf{r}_{12}\right)+N_{d d s}\left(\mathbf{r}_{12}\right), \\
N_{d d}\left(\mathbf{r}_{12}\right)+X_{d d}\left(\mathbf{r}_{12}\right)=N_{d d s}\left(\mathbf{r}_{12}\right)+X_{d d s}\left(\mathbf{r}_{12}\right) .
\end{gathered}
$$

The short-range function $u_{s}\left(\mathbf{r}_{12}\right)$ (going to $-\infty$ for small distances and healing to 0 for large distances) and its longrange counterpart are then chosen as

$$
\begin{aligned}
u_{s}\left(\mathbf{r}_{12}\right)=-2 K_{0}\left(Q\left|\mathbf{r}_{12}-\vec{\alpha}\right|\right)-2 K_{0}\left(Q\left|\mathbf{r}_{12}+\vec{\alpha}\right|\right), & \\
u_{l}\left(\mathbf{r}_{12}\right)= & 2\left[\ln \left(\left|\mathbf{r}_{12}-\vec{\alpha}\right|\right)+K_{0}\left(Q\left|\mathbf{r}_{12}-\vec{\alpha}\right|\right)\right]+2\left[\ln \left(\left|\mathbf{r}_{12}+\vec{\alpha}\right|\right)\right. \\
& \left.+K_{0}\left(Q\left|\mathbf{r}_{12}+\vec{\alpha}\right|\right)\right],
\end{aligned}
$$

where $K_{0}(x)$ is the modified Bessel function, and $Q$ is a cutoff parameter of order 1 . We recall that the 2D FT (Ref. 40) of $u_{l}\left(\mathbf{r}_{12}\right)$ is 


$$
\tilde{u}_{l}(\mathbf{q})=-\frac{4 \pi Q^{2}}{q^{2}\left(q^{2}+Q^{2}\right)}\left(e^{i \mathbf{q} \cdot \vec{\alpha}}+e^{-i \mathbf{q} \cdot \vec{\alpha}}\right) .
$$

The final set of equations is solved by a standard iterative procedure. There is one necessary approximation within the FHNC method in order to obtain a closed set of equations for the nodal and non-nodal functions: a small set of cluster diagrams (corresponding to the so-called elementary diagrams), which cannot be included $a b$ initio in the method, needs to be somehow estimated outside the FHNC method. Several schemes have been devised to include the contribution of such diagrams at various levels of approximation; however, the simplest approximation of totally neglecting these terms (called the FHNC/0 approximation where we assume $E_{d d}=E_{d e}=E_{e e}=E_{c c}=0$ ) generally leads to very reliable results $33,41,42,44$ and we have adopted it in this paper.

\section{RESULTS AND DISCUSSIONS}

In this work we applied the FHNC theory to study the BRS state at filling 1/2 of an arbitrary LL. For the sake of simplicity we neglected the elementary diagrams (FHNC/0). This has allowed us to determine to a reasonable accuracy the pair distribution function and the static structure factor. In order to compare the $\alpha=0$ (RR state, isotropic) with the $\alpha \neq 0$ (BRS state, or nematic) we studied the properties of the BRS wave function for several $\alpha$ 's with magnitudes between 0 and 3 (in general $\alpha=|\alpha| e^{i \theta_{\alpha}}$, but without losing generality we considered only $\left.\theta_{\alpha}=0\right)$.

In Fig. 1 we plot the pair distribution function $g(\mathbf{r})$ for $\alpha=2$ [panels (a) and (b)], and the angle-averaged pair distribution function $\bar{g}(r)$ corresponding to $\alpha=0,1,2,2.5$, and 3 [panels (c) and (d)]. It is interesting to note, for $\alpha$ $\neq 0$, the noticeable angle dependence of $g(\mathbf{r})$, and the splitting of the triple node at the origin to a simple node at the origin and additional simple nodes at $r=\alpha$ and angle $\theta$ $=\theta_{\alpha}, \theta_{\alpha}+\pi\left(\theta_{\alpha}=0\right.$ in this case $)$. From the energetics point of view [see Eq. (3)], a major consideration is the strong dependence of $\bar{g}(r)$ on the value of the parameter $\alpha$. As $\alpha$ is increased the major peak of $\bar{g}(r)$ becomes less pronounced, and for $\alpha \approx 3$ it develops a shoulder for small $r$ [panel (c)]. In addition, note the change in the small- $r$ behavior of $\bar{g}(r)$, which switches from $\propto r^{6}$ (for $\alpha=0$ ) to $\propto r^{2}$ as $\alpha$ is increased [panel (d)]. In general, for small $r, \bar{g}(r)$ has almost no angular dependence, ${ }^{45}$ and for $\alpha \neq 0, g(r \approx 0, \theta)$ $\simeq C_{\alpha} r^{2}$ for $0 \leqslant r \leqslant 0.5$, where $C_{\alpha} \simeq 0.024 \alpha^{1.7}$.

In Fig. 2 we plot the static structure factor $S(\mathbf{q})$ for $\alpha$ $=2$ (top panel), where the most important feature is the emergence of peaks in $S(\mathbf{q})$ characteristic of a nematic structure; and the angle-averaged static structure factor $\bar{S}(q)$ corresponding to $\alpha=0,1,2,2.5$, and 3 (bottom panel). Note the considerable dependence of $\bar{S}(q)$ on $\alpha$ : as it increases the peak is broadened and flattened, with no significant change in the small- $q$ behavior.

One can compute the correlation energy per particle either directly from Eqs. (3), (5), or (8) to determine the energy per electron for arbitrary values of the Fermi BRS parameter $\alpha$, the $2 \mathrm{D}$ system width $\lambda$, and Landau-level index $L$. The following simplified formula can be used in view of Eq. (7):

$$
E_{\alpha}^{L}(\lambda)=\frac{1}{4 \pi} \int_{0}^{\infty} d q q \widetilde{V}(q, \lambda)\left[L_{L}\left(\frac{q^{2}}{2}\right)\right]^{2}[\bar{S}(q)-1],
$$

where $\widetilde{V}(q, \lambda)=\left(2 \pi e^{2} / \epsilon q\right) \exp (-\lambda q)$ is the 2D FT of the ZDS interaction potential. ${ }^{39}$ In addition to allowing straightforward calculations to be extended to any LL, Eq. (20) permits a higher numerical accuracy in the calculation of $E_{\alpha}$ since $\bar{S}(q)$ saturates exponentially to 1 for relatively small values of $q$ as compared to $\bar{g}(r)$.

Figure 3 shows the energy difference between BRS states with $\alpha=1,2,2.5$ and 3, and the isotropic state with $\alpha=0$. Our findings indicate that in the LLL $(L=0)$ the RR state is stable for any $\lambda$, since all $\alpha \neq 0$ states have higher energies (top panel).

The situation changes considerably in higher LL's ( $L$ $\geqslant 1)$. In general, Fermi BRS states are found to have lower energies, and the compressible RR state is unstable toward a nematic state (see the lower panels of Fig. 3). Contrary to our findings for one-third-filled LL's, ${ }^{24}$ we do not see a runaway instability but rather find that there are optimal values: $\alpha_{1}^{*}$ $\approx 2$ for the first $\operatorname{LL}(L=1)$ and $\alpha_{2}^{*} \approx 1$ for $L=2$.

Experiments have not observed a BRS state for the $L$ $=1 \mathrm{LL}$ at filling factors $\nu=5 / 2$ and $7 / 2$. There, remarkable even-denominator FQHE's have been observed, indicating the lack of gapless excitations. It is clear that a gapless compressible Fermi-liquid-like state, such as either RR's state or our BRS state, is less likely to be a good description of the true ground state. Paired CF states ${ }^{37}$ (most likely Pfaffianlike spin-polarized ${ }^{38}$ ) have been proposed to explain these experiments. For higher LL's $(L \geqslant 2)$, however, it is likely that the BRS states proposed here is related to the lowtemperature anisotropic conductance found experimentally. ${ }^{19-23}$

At this point it is important to comment on how precise our determination of these energy differences is. There are two aspects to this problem. First, the reader should note that we used an unprojected wave function in place of the RR generalization [Eq. (2)]. While it would be highly desirable to incorporate this LL projection operators into the formalism, this is not feasible within the FHNC method used here. We should note, however, that the presence of the Jastrow factors in Eq. (2), already provides a considerable projection into the LLL, and is believed to be particularly effective as far as ground-state properties are concerned. ${ }^{43}$ The second aspect is that the FHNC/0 method is essentially a variational method, giving energies that constitute an upper bound to the exact ground-state energy. ${ }^{46}$ While a precise estimate of these errors can only be made a posteriori by comparing these results with alternative calculations, these methods have proven to be quite reliable in scenarios ranging from other partially filled LL's (Refs. 24 and 42) to ${ }^{3} \mathrm{He}$ systems. ${ }^{33,41}$ While the energy differences we are interested in (see Fig. 3) are quite small, and perhaps smaller than the errors in the absolute values of the correlation energy in the 

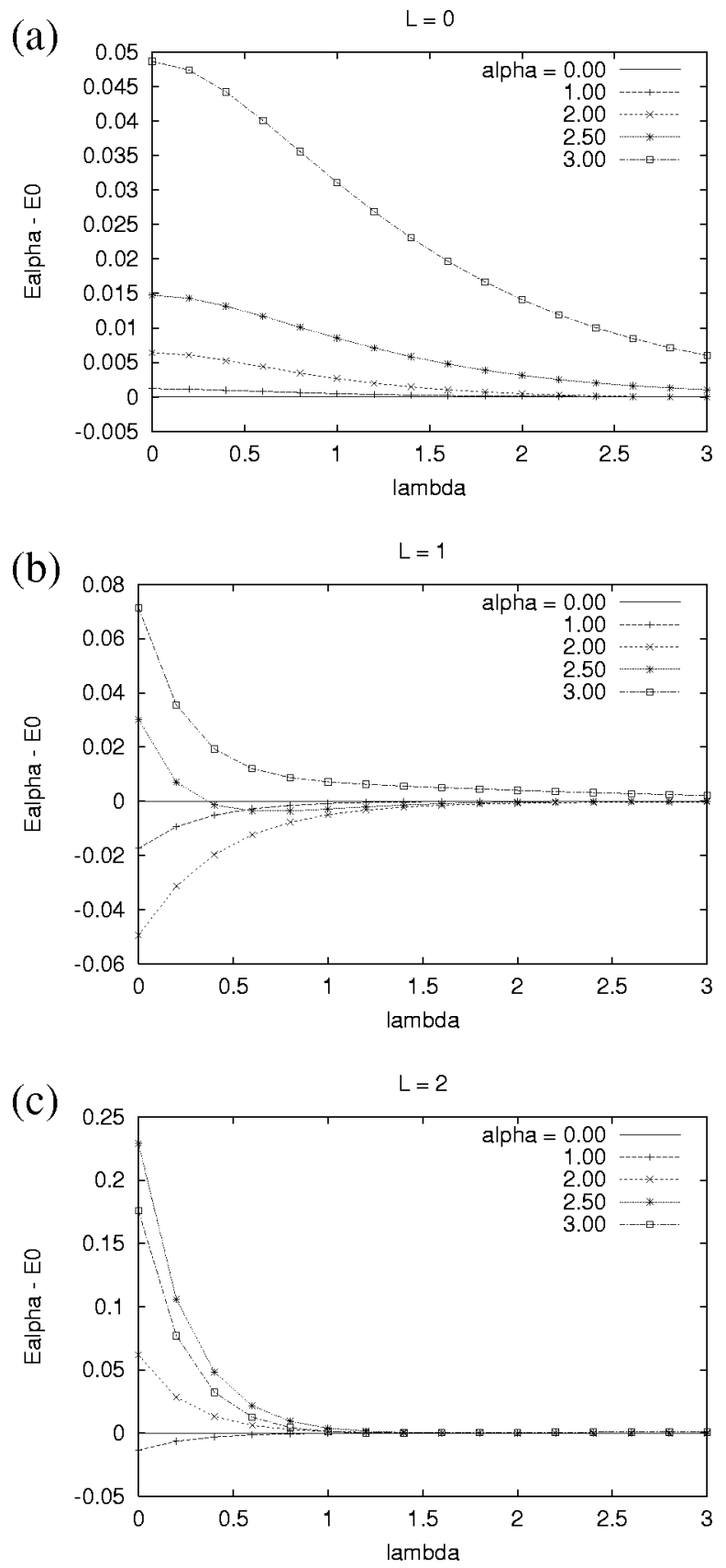

FIG. 3. Energy per particle of the Fermi BRS states with $\alpha$ $=1,2,2.5$, and 3 relative to the isotropic $(\alpha=0)$ state: $\Delta E_{\alpha}(\lambda)$ $=E_{\alpha}(\lambda)-E_{0}(\lambda)$ for the LLL $(L=0)$ and higher LL's as functions of the short-distance cutoff parameter $\lambda$. Energies are in units of $e^{2} /\left(\epsilon l_{o}\right)$. Note that in the LLL, the Fermi BRS states always have an energy higher than the isotropic state, whereas in higher LL's $(L=1,2)$ there are ranges of $\lambda$ for which Fermi BRS states are favorable.

states, we remark that these are not uncorrelated errors but systematic deviations due to the nature of the approximations used, and energy differences will likely be considerably more precise.

In conclusion, we applied the FHNC theory to study possible Fermi BRS states in a half-filled LL. We find that the isotropic RR state is stable in the LLL for realistic interaction potentials. In higher LL's, BRS states with nematic order are energetically more favorable than the RR state, perhaps with a direct connection to the anisotropic states observed recently in high LL's. ${ }^{19-21}$

\section{ACKNOWLEDGMENTS}

We would like to acknowledge helpful discussions with A. T. Dorsey, M. Fogler, L. Radzihovsky, and G. Vignale. This work was supported by the University of Missouri Research Board.

\section{APPENDIX}

For a Fermi system at density $\rho$ and spin degeneracy $g_{s}(1$ or 2 ) the sum of nonnodal (composite) diagrams is given by

$$
\begin{aligned}
& X_{d d}\left(\mathbf{r}_{12}\right)=e^{u\left(\mathbf{r}_{12}\right)+N_{d d}\left(\mathbf{r}_{12}\right)+E_{d d}\left(\mathbf{r}_{12}\right)}-N_{d d}\left(\mathbf{r}_{12}\right)-1, \\
& X_{d e}\left(\mathbf{r}_{12}\right)=e^{u\left(\mathbf{r}_{12}\right)+N_{d d}\left(\mathbf{r}_{12}\right)+E_{d d}\left(\mathbf{r}_{12}\right)}\left[N_{d e}\left(\mathbf{r}_{12}\right)+E_{d e}\left(\mathbf{r}_{12}\right)\right] \\
& -N_{d e}\left(\mathbf{r}_{12}\right) \\
& X_{e e}\left(\mathbf{r}_{12}\right)=e^{u\left(\mathbf{r}_{12}\right)+N_{d d}\left(\mathbf{r}_{12}\right)+E_{d d}\left(\mathbf{r}_{12}\right)}\left[N_{e e}\left(\mathbf{r}_{12}\right)+E_{e e}\left(\mathbf{r}_{12}\right)\right. \\
& +\left|N_{d e}\left(\mathbf{r}_{12}\right)+E_{d e}\left(\mathbf{r}_{12}\right)\right|^{2}-g_{s} \mid N_{c c}\left(\mathbf{r}_{12}\right)+E_{c c}\left(\mathbf{r}_{12}\right) \\
& \left.-l\left(\mathbf{r}_{12}\right) /\left.g_{s}\right|^{2}\right]-N_{e e}\left(\mathbf{r}_{12}\right) \text {, } \\
& X_{c c}\left(\mathbf{r}_{12}\right)=e^{u\left(\mathbf{r}_{12}\right)+N_{d d}\left(\mathbf{r}_{12}\right)+E_{d d}\left(\mathbf{r}_{12}\right)}\left[N_{c c}\left(\mathbf{r}_{12}\right)+E_{c c}\left(\mathbf{r}_{12}\right)\right. \\
& \left.-l\left(\mathbf{r}_{12}\right) / g_{s}\right]+l\left(\mathbf{r}_{12}\right) / g_{s}-N_{c c}\left(\mathbf{r}_{12}\right) \text {. }
\end{aligned}
$$

The chain formation of the nodal diagrams is generated by convolution equations

$$
N_{d d}\left(\mathbf{r}_{12}\right)=\rho \int d \mathbf{r}_{3}\left[X_{d d}\left(\mathbf{r}_{13}\right)+N_{d d}\left(\mathbf{r}_{13}\right)\right] P\left(\mathbf{r}_{32}\right)
$$

$$
\begin{aligned}
N_{d e}\left(\mathbf{r}_{12}\right)= & \rho \int d \mathbf{r}_{3}\left[X_{d d}\left(\mathbf{r}_{13}\right) X_{e e}\left(\mathbf{r}_{32}\right)-X_{d e}\left(\mathbf{r}_{13}\right) X_{d e}\left(\mathbf{r}_{32}\right)\right. \\
& \left.+\left[X_{d e}\left(\mathbf{r}_{13}\right)+N_{d e}\left(\mathbf{r}_{13}\right)\right] P\left(\mathbf{r}_{32}\right)\right], \\
N_{e e}\left(\mathbf{r}_{12}\right)= & \rho \int d \mathbf{r}_{3}\left[X_{d e}\left(\mathbf{r}_{13}\right) X_{d e}\left(\mathbf{r}_{32}\right)-X_{d d}\left(\mathbf{r}_{13}\right) X_{e e}\left(\mathbf{r}_{32}\right)\right. \\
& \left.+\left[X_{e e}\left(\mathbf{r}_{13}\right)+N_{e e}\left(\mathbf{r}_{13}\right)\right] P\left(\mathbf{r}_{32}\right)\right], \\
N_{c c}\left(\mathbf{r}_{12}\right)= & \rho \int d \mathbf{r}_{3}\left[-l\left(\mathbf{r}_{13}\right) / g_{s}+X_{c c}\left(\mathbf{r}_{13}\right)\right. \\
& \left.+N_{c c}\left(\mathbf{r}_{13}\right)\right] X_{c c}\left(\mathbf{r}_{32}\right),
\end{aligned}
$$


where

$$
\begin{aligned}
P\left(\mathbf{r}_{i j}\right)= & X_{d d}\left(\mathbf{r}_{i j}\right)+2 X_{d e}\left(\mathbf{r}_{i j}\right)+\rho \int d \mathbf{r}_{k}\left[X_{d d}\left(\mathbf{r}_{i k}\right) X_{e e}\left(\mathbf{r}_{k j}\right)\right. \\
& \left.-X_{d e}\left(\mathbf{r}_{i k}\right) X_{d e}\left(\mathbf{r}_{k j}\right)\right] .
\end{aligned}
$$

The pair distribution function is then given from

$$
\begin{aligned}
g\left(\mathbf{r}_{12}\right)= & 1+X_{d d}\left(\mathbf{r}_{12}\right)+N_{d d}(\mathbf{r} 12)+2\left[X_{d e}\left(\mathbf{r}_{12}\right)+N_{d e}\left(\mathbf{r}_{12}\right)\right] \\
& +X_{e e}\left(\mathbf{r}_{12}\right)+N_{e e}\left(\mathbf{r}_{12}\right) .
\end{aligned}
$$

${ }^{1}$ K. von Klitzing, G. Dorda, and M. Pepper, Phys. Rev. Lett. 45, 494 (1980).

${ }^{2}$ D. C. Tsui, H. L. Stormer, and A. C. Gossard, Phys. Rev. Lett. 48, 1559 (1982).

${ }^{3}$ Perspectives in Quantum Hall Effects, edited by S. Das Sarma and A. Pinczuk (Wiley, New York 1996).

${ }^{4}$ R. B. Laughlin, Phys. Rev. Lett. 50, 1395 (1983).

${ }^{5}$ D. J. Thouless, Topological Quantum Numbers in Nonrelativistic Physics (World Scientific, Singapore, 1998).

${ }^{6}$ X. G. Wen, Phys. Rev. B 43, 11025 (1991); Phys. Rev. Lett. 64, 2206 (1990).

${ }^{7}$ For a review, see J. Jain, Phys. Today 53(4), 39 (2000); and/or Composite Fermions, edited by O. Heinonen (World Scientific, New York, 1998).

${ }^{8}$ R. L. Willett, J. P. Eisenstein, H. L. Stormer, D. C. Tsui, A. C. Gossard, and J. H. English, Phys. Rev. Lett. 59, 1776 (1987).

${ }^{9}$ R. L. Willett, M. A. Paalanen, R. R. Ruel, K. W. West, L. N. Pfeiffer, and D. J. Bishop, Phys. Rev. Lett. 65, 112 (1990).

${ }^{10}$ F. D. M. Haldane, Phys. Rev. Lett. 55, 2095 (1985).

${ }^{11}$ For a review of experimental evidence for the filling factor $\nu$ $=1 / 2$ see H. L. Stormer and D. C. Tsui, in Perspectives in Quantum Hall Effects (Ref. 3).

${ }^{12}$ R. R. Du, H. L. Stormer, D. C. Tsui, L. N. Pfeiffer, and K. W. West, Solid State Commun. 90, 71 (1994).

${ }^{13}$ V. J. Goldman, B. Su, and J. K. Jain, Phys. Rev. Lett. 72, 2065 (1994).

${ }^{14}$ B. I. Halperin, P. A. Lee, and N. Read, Phys. Rev. B 47, 7312 (1993).

${ }^{15}$ J. K. Jain, Phys. Rev. Lett. 63, 199 (1989).

${ }^{16}$ E. Rezayi and N. Read, Phys. Rev. Lett. 72, 900 (1994).

${ }^{17}$ R. L. Willett, J. P. Eisenstein, H. L. Stormer, D. C. Tsui, A. C. Gossard, and J. H. English, Phys. Rev. Lett. 59, 1776 (1987); W. Pan, J.-S. Xia, V. Shvarts, D. E. Adams, H. L. Stormer, D. C. Tsui, L. N. Pfeiffer, K. W. Baldwin, and K. W. West, Phys. Rev. Lett. 83, 3530 (1999).

${ }^{18}$ See, e.g., V. W. Scarola, K. Park, and J. K. Jain, Nature (London) 406, 863 (2000), and references therein; N. Read, cond-mat/0010071 (unpublished); Physica B 298, 121 (2001), and references therein.

${ }^{19}$ M. P. Lilly, K. B. Cooper, J. P. Eisenstein, L. N. Pfeiffer, and K. W. West, Phys. Rev. Lett. 82, 394 (1999).

${ }^{20}$ R. R. Du, D. C. Tsui, H. L. Stormer, L. N. Pfeiffer, K. W. Baldwin, and K. W. West, Solid State Commun. 109, 389 (1999).

${ }^{21}$ M. Shayegan, H. C. Manoharan, S. J. Papadakis, and E. P. DePoortere, Physica E 6, 40 (2000).
${ }^{22}$ E. Fradkin and S. A. Kivelson, Phys. Rev. B 59, 8065 (1999).

${ }^{23}$ C. Wexler and A. T. Dorsey, Phys. Rev. B 64, 115312 (2001).

${ }^{24}$ O. Ciftja and C. Wexler, Phys. Rev. B 65, 045306 (2002).

25 A. A. Koulakov, M. M. Fogler, and B. I. Shklovskii, Phys. Rev. Lett. 76, 499 (1996).

26 M. M. Fogler, A. A. Koulakov, and B. I. Shklovskii, Phys. Rev. B 54, 1853 (1996).

27 R. Moessner and J. T. Chalker, Phys. Rev. B 54, 5006 (1996).

28 T. Jungwirth, A. H. MacDonald, L. Smrčka, and S. M. Girvin, Phys. Rev. B 60, 15574 (1999).

${ }^{29}$ Also see A. H. MacDonald and M. P. A. Fisher, Phys. Rev. B 61, 5724 (2000); H. Yi, H. A. Fertig, and R. Côté, Phys. Rev. Lett. 85, 4156 (2000).

${ }^{30}$ K. Musaelian and R. Joynt, J. Phys.: Condens. Matter 8, L105 (1996).

${ }^{31}$ S. Fantoni and S. Rosati, Nuovo Cimento Lett. 10, 545 (1974); S. Fantoni and S. Rosati, Nuovo Cimento A 25, 593 (1975).

${ }^{32}$ E. Krotscheck and M. L. Ristig, Phys. Lett. 48A, 17 (1974); Nucl. Phys. A 242, 389 (1975).

${ }^{33}$ J. G. Zabolitzky, Phys. Rev. A 16, 1258 (1977).

${ }^{34}$ E. Manousakis, S. Fantoni, V. R. Pandharipande, and Q. N. Usmani, Phys. Rev. B 28, 3770 (1983).

${ }^{35}$ M. L. Ristig and J. W. Clark, Phys. Rev. B 14, 2875 (1976).

${ }^{36}$ J. Bardeen, L. N. Cooper, and J. R. Schrieffer, Phys. Rev. 106, 162 (1957).

${ }^{37}$ G. Moore and N. Read, Nucl. Phys. B 360, 362 (1991).

${ }^{38}$ R. H. Morf, Phys. Rev. Lett. 80, 1505 (1998).

${ }^{39}$ F. C. Zhang and S. Das Sarma, Phys. Rev. B 33, 2903 (1986).

${ }^{40}$ We use the standard convention for the 2D FT: $\widetilde{f}(\mathbf{q})=\int d^{2} r \exp$ $[-i \mathbf{q} \cdot \mathbf{r}] f(\mathbf{r})$, and $f(\mathbf{r})=\int d^{2} q /(2 \pi)^{2} \exp [i \mathbf{q} \cdot \mathbf{r}] \widetilde{f}(\mathbf{q})$.

${ }^{41}$ J. G. Zabolitzky, Phys. Lett. 64B, 233 (1976).

${ }^{42}$ O. Ciftja and S. Fantoni, Phys. Rev. B 56, 13290 (1997).

${ }^{43}$ N. Trivedi and J. K. Jain, Mod. Phys. Lett. B 5, 503 (1993).

${ }^{44}$ O. Ciftja, S. Fantoni, J. W. Kim, and M. L. Ristig, J. Low Temp. Phys. 108, 357 (1997).

${ }^{45}$ The absence of angular dependence on $g(r, \theta)$ for small $r$ can be easily understood by noting that, in the small- $r$ limit, $g(r$ $\approx 0, \theta) \propto \exp \left[u_{s}(r \approx 0, \theta)\right]\left[1-l(r \approx 0)^{2} / g_{s}\right], \quad\left(g_{s}=1\right) \quad$ where $u_{s}(r, \theta)$ is given from Eq. (17). The second term in the above expression is angle independent, and represents the zero of the Pauli principle. For short distances $\lim _{r \rightarrow 0} K_{0}(Q r)=-\ln (Q r / 2)$ $-\gamma, \quad(\gamma=0.5772 \ldots \quad$ is Euler's constant $) ;$ therefore, $u_{s}(r$ $\approx 0, \theta)]$ and, by consequence $g(r \approx 0, \theta)$, is angle independent in this region. For more details see Ref. 24.

${ }^{46}$ G. Ripka, Phys. Rep. 56, 1 (1979). 